\title{
Applications of Simple Nanoparticle Zeolitic Imidazolate Framework-8 in Biomedicine
}

\author{
Zhaokun Zhang, ${ }^{\dagger, a}$ Yan Xu, ${ }^{\dagger, *, b}$ Kunfeng Zhao, ${ }^{*, a, b}$ Ying Zhang, ${ }^{b}$ Weijia Chen, ${ }^{c}$ Xiaodi Li, ${ }^{d}$ Yanxia Meng, ${ }^{a}$ \\ Dicheng Yang, ${ }^{b}$ Ping Wang, ${ }^{b}$ and Jun Zhu ${ }^{\dagger, *, a, b}$ \\ a School of Medical Instrument and Food Engineering, University of Shanghai for Science and Technology, \\ Shanghai 200093, China \\ ${ }^{b}$ National Engineering Research Center for Nanotechnology, Shanghai 200241, China \\ ${ }^{c}$ School of Materials Science and Engineering, Shanghai Jiao Tong University, Shanghai 200240, China \\ ${ }^{d}$ Shanghai University of Traditional Chinese Medicine, Shanghai 201210, China
}

Email: yxusinap@163.com (Y. X.), zhaokunfeng-gl@163.com (K. Z.),yzjzhu@163.com (J. Z.).

${ }^{\dagger}$ These authors contributed equally to this work.

\begin{abstract}
Zeolitic Imidazolate Framework-8 (ZIF-8) is a type of typical metal organic framework (MOF), which is an emerging porous coordination polymers that is bridged by zinc metal ions and organic linkages. Due to its intrinsic porous characteristics, unique $\mathrm{pH}$ sensitivity as well as superior drug loading efficiency, zeolitic imidazolate framework- 8 has attracted extensive attention and received a wide range of applications in the field of biomedicine. In this review, we briefly discuss the effective delivery and controlled release of various drugs in ZIF-8, such as functional proteins, hydrophilic and hydrophobic drugs. Understanding the processes of various drugs loading is important for developing new drug delivery systems. In addition, we also explored the cooperation mechanism between ZIF and different materials. The combination of chemotherapy, photodynamic therapy, photothermal therapy and medical imaging have been applied in the field of nanomedicine and have made emarkable achievements.
\end{abstract}

Keywords zeolitic imidazolate framework-8, delivery and controlled release, multifunctional nano-platform

\section{Introduction}

Zeolitic imidazolate frameworks (ZIFs) are a new and special class of porous crystals with extended tetrahedral topologies consist of M-Im-M (where M stands for $\mathrm{Zn}$, Co cation and Im stands for imidazolate anions) formed by a self-assembly approach, with the M-Im-M angle around $145^{\circ} .^{[1-4]}$ ZIFs possess diverse tetrahedral topologies structures (e.g., ZIF-3, ZIF-8, ZIF-20, ZIF-67, Figure 1). Due to their intrinsic porous characteristics, abundant functionalities as well as exceptional thermal and chemical stabilities, ZIFs have widely applications in gas separation, catalysis, sensing and electronic devices, and drug delivery. ${ }^{[5-10]}$

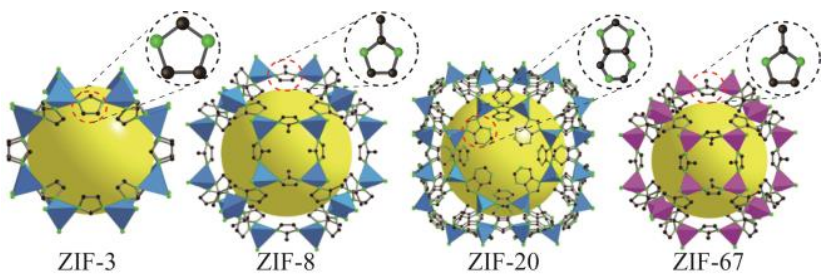

Figure 1 Crystal structures of ZIF-3, ZIF-8, ZIF-20, ZIF-67. The largest cage is shown with $\mathrm{ZnN}_{4}$ in blue polyhedra and $\mathrm{CoN}_{4}$ in pink polyhedra, and the links in ball-and-stick presentation. The yellow ball indicates space in the cage. $\mathrm{H}$ atoms are omitted for clarity (C, black; N, green).

Noteworthy, unlike other ZIFs, zeolitic imidazolate framework-8 (ZIF-8) is bridged by zinc metal ions and organic linkages, which has better $\mathrm{pH}$ sensitivity and drug loading capacity and have superior performance in applications of biomedicine.
ZIF-8 has proven to be an efficient nanocarrier for medical imaging and drug delivery. In order to achieve the desired physicochemical properties, ZIF-8 can be produced with controllable sizes and shapes. ${ }^{[11-15]}$ Moreover, with the large specific surface area and high porosity, ZIF-8 can achieve a relative high drug loading capacity. Afterwards, weak coordination bonds between $\mathrm{Zn}$ and Im ensure that the ZIF-8 is easily biodegradable in tumor microenvironment. The unique frame structure provides ZIF-8 with exceptional thermal stability. ${ }^{[16]}$ It is worth noting that in the micro-acid environment of the tumor, the $\mathrm{pH}$ sensitivity of ZIF-8 further satisfies the requirement for rapid release of loading drugs from nanomedicine carriers. ${ }^{[17-20]}$ Utilizing these advantages, ZIF-8 nanoparticles are widely used as drug nanocarriers for delivering proteins and small-molecule chemicals (Table 1). ${ }^{[21-33]}$ In addition, ZIF-8 drug delivery systems are good candidates to construct the multifunctional nanoplatforms for diagnosis and treatments, and further exert its great potential in the biomedical field such as easily modified composite carriers and medical imaging. In summary, these desirable characteristics enable ZIF-8 to be promising platforms for drug delivery, clinical tumor therapy, and other disease treatments. ${ }^{[34-36]}$

This review will highlight developments of ZIF-8 in the fields of drug delivery, multifunctional cancer therapy, and medical imaging, including the scope of drug loading and different structural granules. The prospects and challenges of ZIF-8 in drug therapy, photothermal therapy and photodynamic therapy are also addressed. Finally, the challenges of using ZIF-8 for better cancer treatment and addressing future clinical application are discussed (Figure 2). Compared with other excellent reviews that discuss the various applications of MOFs, the 
Table 1 Examples of ZIF-8 as drug nanocarriers

\begin{tabular}{|c|c|c|c|c|}
\hline Other important components ${ }^{a}$ & Drug/cargo & Loading percentage/wt\% & Targeted cell lines & Ref. \\
\hline PVP & BSA, $\beta$-Gal, caspase 3, HSA & - & HeLa, Hacat, Skvo3 & [21] \\
\hline Ara & IR820 & 61.2 & $4 \mathrm{~T} 1$ & [22] \\
\hline PEG-PUSeSe-PEG & DOX & 47.2 & MDA-MB-231 & [23] \\
\hline Indocyanine Green (ICG) & DOX & - & L929 & [24] \\
\hline EGCG, Fe(III) & DOX, $\mathrm{H}_{2} \mathrm{O}_{2}$ & - & B16, BEL-7402, SMC & [25] \\
\hline CD, PdNS, & PAA, DOX & - & HepG-2, A549, MCF-7 & [26] \\
\hline $\mathrm{MnO}_{2}$ & $\mathrm{C}_{3} \mathrm{~N}_{4}, \mathrm{DOX}$ & - & $4 \mathrm{~T} 1$ & [27] \\
\hline Cell membrane & CAT, DOX & - & B16F10, HepG2, COS7 & [28] \\
\hline Erythrocyte & GOx, TPZ & - & СT26, Macrophages & [29] \\
\hline- & 3-MA & 19.798 & Hela & [30] \\
\hline- & CRISPR/Cas 9 & 17.0 & $\mathrm{CHO}$ cells & [31] \\
\hline- & $\mathrm{ZnPc}$ & - & HepG-2 cells & [32] \\
\hline CpG ODNs & OVA & - & RAW264.7 & [33] \\
\hline
\end{tabular}

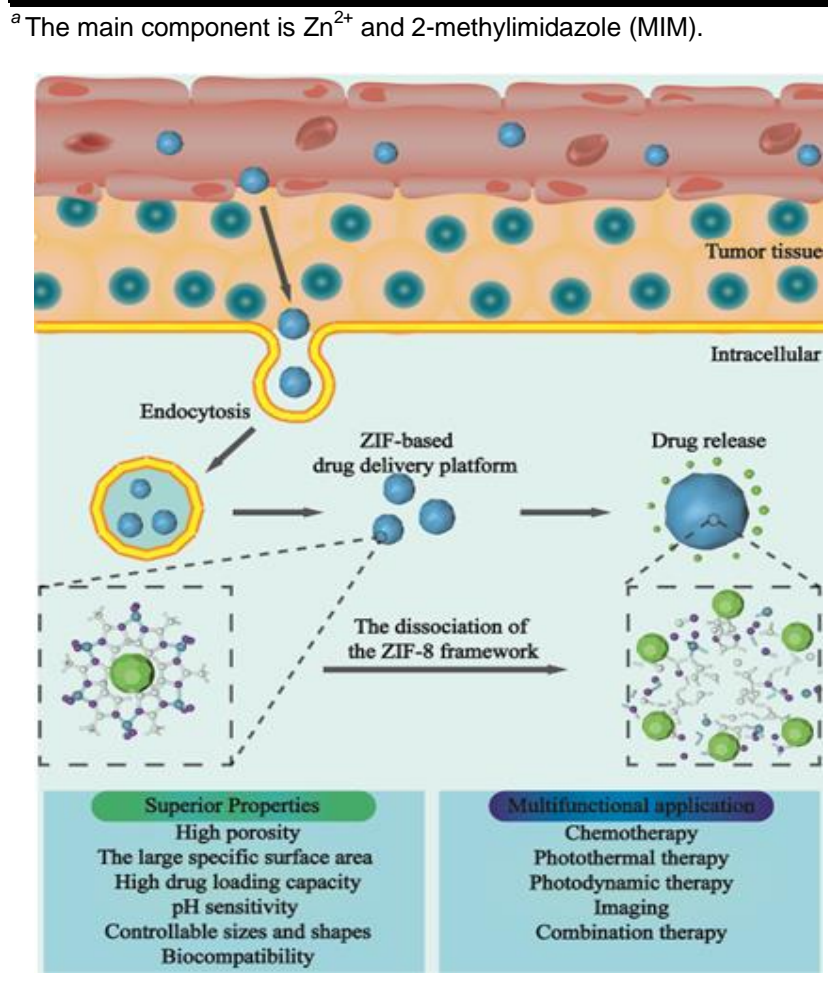

Figure 2 Schematic illustration of ZIF-8-based system for drug delivery and application.

difference in this evaluation is that it will focus on the bio-application of ZIF-8, from drug carriers to different structural cancer treatment platforms, and the development of ZIF-8 in medical imaging and its potential clinical application. ${ }^{[37-39]}$

\section{Delivery and Controlled Release of Different Cargos}

The rapid development of nanomaterials provides an opportunity to overcome challenges of drug delivery in the medical field. Inspired by the large surface areas, well-defined structures, and highly ordered porosities, Sun et al. found that ZIF-8 was suitable candidate to be used as an effective drug delivery vehicle. ${ }^{[40]}$ Therefore, the interactions between ZIF-8 and biomacromolecule and organisms are firstly explored to elucidate the biocompatibility and protectivity of ZIF-8.

Biomarcomolecules and living organisms are sensitive to their surrounding environments, and easily to be biologically inactivated to subtle changes of salinity, $\mathrm{pH}$, temperature, pressure, moisture and nutrients. Strategies were proposed to design bioactive nanocoatings to maintain activity, for example, polymers, mesoporous silica, liposome and MOF. Lyu et al. established a cytochrome c/ZIF-8(Cyt c/ZIF-8) composite through one-pot synthesis, and discovered the peroxidase activity of Cyt c embedded in ZIF-8 10 times higher than that of free Cyt $\mathrm{c}$. This work demonstrated the fireworks formed by ZIF-8 and enzyme creating a suitable microenvironment for enzyme catalysis with an increased affinity towards substrate. ${ }^{[41]}$ Furthermore, tabacco mosaic virus (TMV) was coated with ZIF-8 MOF to construct size and morphology tunable TMV@ZIF NPs, resulting in extended stability in extreme environments without losing its surface functionalizability. Afterwards, Falcaro et al. synthesized a $\beta$-galactosidase ( $\beta$-gal)/ ZIF-8 shell to coat yeast cells, and discovered bioactive exogenous enzymes could provide cells with nutrients to survive in the environments of toxic agents and UV irradiation. ${ }^{[42]}$ These works confirmed the potential of ZIF-8 MOFs applying for biomineralization, supplying supportable microenvironments to alleviate damages caused by external factors.

Having evaluated the active conservation of biomolecules supported by ZIF-8 MOFs, we then focus on their ability of protein delivery and controlled release. Efficient delivery of functional proteins in living cells remains a huge challenge in protein therapy for native protein are prone to degradation by cellular enzymes. ${ }^{[43-45]}$ To solve this problem, Chen et al. reported a highly efficient protein delivery platform for controlled protein release based on the ZIF-8 nanoparticles. They encapsulated proteins in ZIF-8 nanocarrier and modified the nanoparticles using a polyvinylpyrrolidone (PVP) coating to offer nanocarriers sufficient stability in cell culture medium (Figure 3A) ${ }^{[21]}$ In vitro results indicated the nanoparticles with a high loading of proteins could maintain protein activity for several months, and protect proteins from enzyme-mediated degradation. Furthermore, the nanocarriers were effective in releasing proteins and escaping from internal lysosomes, and maintaining protein activity in living cells (Figure 3B). Interestingly, this work afforded a useful strategy of protein-encap- 
A

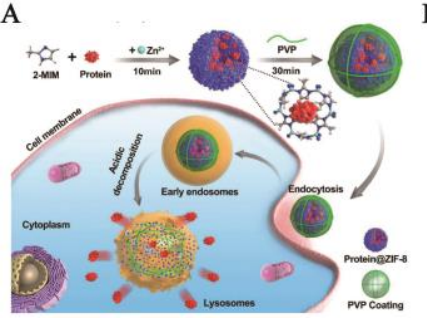

B

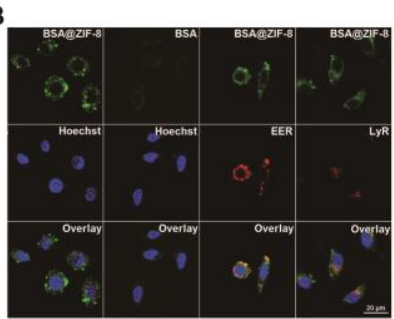

Figure 3 A) Illustration of biomineralized MOF NPs for protein delivery in living cells. B) Delivery and endo-lysosomal escape in HeLa cells of FITC-labeled BSA in PVP-coated ZIF-8 NPs.

sulating MOFs for effective protein delivery, rapid release, escaping from lysosome and enzyme-mediated degradation. Loading of various drugs in ZIF-8 were also explored. ZIF-8 achieved high-load loading of some drugs with unique functional groups, drugs with unique functional groups $(\mathrm{COOH}$, $\mathrm{SO}_{3} \mathrm{H}, \mathrm{C}=\mathrm{O}$, etc.) could strongly interact with $\mathrm{ZIF}-8$ and easily be in-situ encapsulated. ${ }^{[11,46,47]}$ For example, ZIF- 8 has been proven high loading efficiency of anti-cancer drugs doxorubicin hydrochloride (DOX), 5-fluorouracil (5-FU), hydroxyurea and mercaptopurine. ${ }^{[48,49]}$ However, it is also critical to achieve satisfactory drug loading and drug release behavior for drugs without these functional groups.

Recently, Zhang and co-workers proposed a prodrug strategy to solve the problem that loaded drugs without related functional groups (Figures $4 \mathrm{~A}$ and $4 \mathrm{~B}$ ). ${ }^{[22]}$ The cytarabine (Ara) was selected as a model drug, as Ara was difficult to load intoZIF-8 vector due to the leakage resulted from its small molecular structure and weak interaction with ZIF-8. Indocyanine green (IR820) was used to bind to Ara for the formation of prodrug (Ara-IR820). Furthermore, sulfonic groups enhanced strong interaction between the prodrug and ZIF-8, and this prodrug loaded ZIF-8 is further functionalized with hyaluronic acid (HA) to produce active targeting HA/Ara-IR820@ZIF-8 nanoparticles (Figures $4 \mathrm{~A}$ and $4 \mathrm{~B}$ ). In vitro and in vivo results of the nanomedicine demonstrated effective targeting ability and $\mathrm{pH}$ responsive drug release behavior, combined with imaging-directed photothermal therapy to cancer treatment. HA/ Ara-IR820@ZIF-8 nanoparticle could accumulate at the tumor area of tumor bearing mice in $1 \mathrm{~h}$, which was more quickly than Ara-IR820@ZIF-8 (Figures 4C and 4D). This strategy addressed a new approach of loading common drugs without strong interaction with MOFs based on the ZIF-8 vector and expands the potential of ZIF-8 as universal drug carriers.

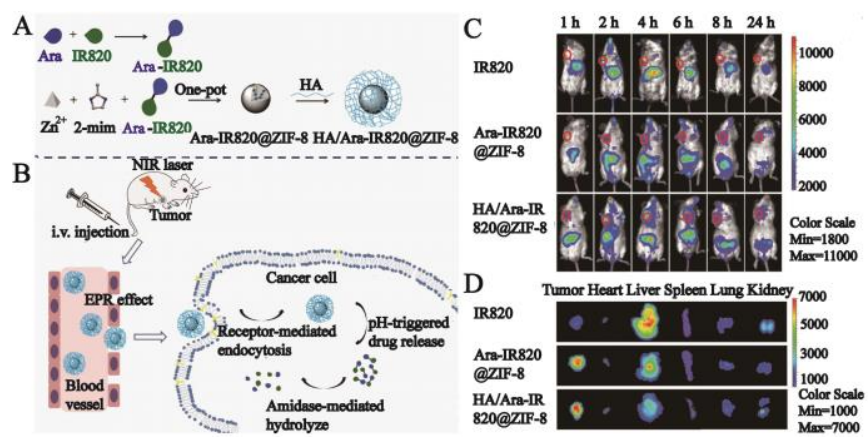

Figure 4 Illustration of prodrug-loaded ZIF-8 for cancer therapy: A)construction of HA/Ara-IR820@ZIF-8 and B) tumortargeted chemo-photothermal therapy. C) The distribution of IR820, Ara-IR820@ZIF-8, and HA/Ara-IR820@ZIF-8 in tumorbearing mice at different times after the intravenous injection. D) Fluorescence imaging of isolated organs and tumors at $24 \mathrm{~h}$ after the treatment with IR820, Ara-IR820 @ZIF-8, and HA/AraIR820@ZIF-8.

Drug delivery systems (DDSs) with controlled release to targeted cancer cells have attracted widespread attention and developing safer and accurate DDSs without interfering with normal cells have become a research hot-spot. Tumor acidic microenvironment has been used as a trigger to direct drug release. More importantly, $\mathrm{pH}$ sensitivity of ZIF-8 plays an important role in stimulating drug release. ${ }^{[50-52]}$ Zhou and co-workers developed a simple and environmentally friendly strategy, in which selenium-containing polymers were encapsulated in ZIF-8 along with doxorubicin (DOX). ${ }^{[23]}$ 2-Methylimidazole was mixed with DOX@P, and then coordinated with $\mathrm{Zn}^{2+}$ ions to form ZIF-8 on the surface of the micelle, denoted as P@ZIF-8. SEM and TEM analysis (Figure 5A) indicated that P@ZIF-8 NPs were dodecahedral shapes and the average diameter was about 200 nm. Moreover, P@ZIF-8 was observed with no obvious core-shell structure. Drug release was achieved though slowly diffusing of cellular redox agent into MOF channel, interacting with micelles of selenium-containing polymer and inducing the decomposition of the micelles, then destroying the core-shell nanostructures and expanding the DOX release. In addition, ZIF-8 degraded in cellular acidulous environment, allowing further releasement of drugs and exhibiting varied drug release pathways towards different external stimulants. In vitro results demonstrated excellent drug-loading and release ability of the prepared P@ZIF-8, and the nanocarrier was suitable for storage and release as an intelligent DDS (Figure 5B). This drug system is highly promising for achieving controlled drug delivery in tumor tissue. The $\mathrm{pH}$ sensitivity of ZIF-8 paves the way for the use of multi-target drug delivery in diagnostics and therapy. Other attempts also have been made to encapsulate drugs in addition to the delivery and controlled release of the above special materials. For example, Feng group proposed a rapid coating strategy based on ZIF-8 to effectively protect antibodies from the impact of outside interference environment. ${ }^{[53]}$ Here, the broad combined use of ZIF-8 and biopharmaceuticals increases the stability and effective release of drug.

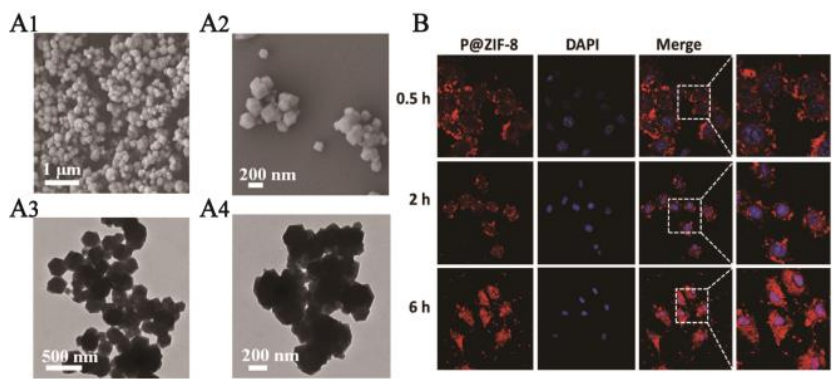

Figure 5 A1, A2) SEM and A3, A4) TEM images of the as-prepared P@ZIF-8. B) Confocal microscopy images of MDA-MB-231 cells incubated with P@ZIF-8 nanocomposites for $0.5,2$, and $6 \mathrm{~h}$. For each panel, the images from left to right show DOX fluorescence in cells (red), cell nuclei stained by DAPI (blue), and overlays of the two images.

\section{Construction of Multifunctional Nano-platform for Cancer Theranostics}

Although cancer diagnosis and treatment methods have been systematic studied, single diagnostic and treatment strategy is greatly limited by its therapeutic effect, and its anti-tumor effect has room for improvement. In order to achieve the desired diagnostic and treatment results, considerable efforts have been made to build multifunctional nanomedicine platforms. ${ }^{[54-56]}$ Among them, ZIF-8-based multifunctional diagnostic and treatment system has significantly increased curative effect of cancer, mainly by combining precise treatment, chemotherapy, photothermal therapy and medical imaging to 
maximize its therapeutic potential. In this section, we will focus on multi-functional nano-platforms based on ZIF-8 nanocarriers, including novel composite structures, cell membrane coatings (precise treatment), and medical imaging.

Adding effective functions is the requisite element for constructing a multifunctional nanocomposite structure. In this way, ZIF-8 NPs have been considered as an ideal choice due to simple synthesis and easy functionalization, especially as a carrier for drugs of different physicochemical properties. Zhang and co-workers reported a unidentified flying object (UFO)-like cyclodextrin-palladium nanosheet/ZIF-8 Janus nanoparticle (CD-PdNS/ZIF-8 JNP) for synergistic dual-drug chemotherapy and photothermal therapy. ${ }^{[26]}$ The novel UFO-like CD-PdNS/ ZIF-8 JNPs prepared via a selective coating of polyacrylic acid (PAA) on one of the flat surfaces of 2D PdNS followed by further formation of ZIF-8 on a PAA template, and alternative modification of CDs on exposed PdNS surface are designed. Figure $6 \mathrm{~A}$ illustrates the preparation and application of the UFO-like CD-PdNS/ZIF-8 JNPs. Polyacrylic acid (PAA), aqueous ammonium hydroxide $\left(\mathrm{NH}_{3} \cdot \mathrm{H}_{2} \mathrm{O}\right)$, and isopropanol (IPA) were added into the obtained PdNS solution in order to form a template for ZIF-8 growth, then carrying the hydrophilic drugs. The CDs carries a lot of hydroxyl groups and possess welldefined hydrophobic microenvironment inside of the cup, which allow to package hydrophobic drugs in their chambers. TEM and SEM images show that the CD-PdNS/ZIF-8 JNPs possess unique composite structures, reasonably sized and uniform (Figure 6B). In vitro and in vivo results indicate that the dual-drug loaded particles are further used synergistic hydrophobic and hydrophilic drug chemotherapy and photothermal cancer therapy (combination index $=0.65$ ). The dual drug-loaded JNP in the laser irradiation group has the highest cancer treatment efficiency. The good performance is due to the synergistic chemotherapy and photothermal therapy in NIR-II biowindow. This work significantly broadens the application of
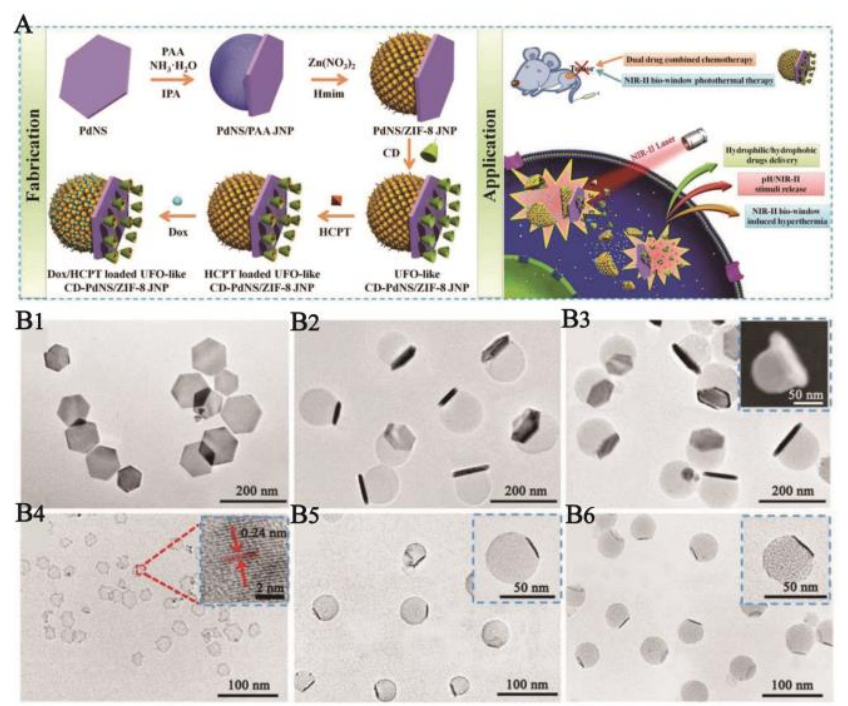

Figure 6 A) Schematic diagram of preparing UFO-like CD-PdNS/ZIF-8 JNPs for in vitro and in vivo $\mathrm{pH}$ and NIR-II (1064 nm) dual-triggered synergistic, dual-drug chemotherapy, and photothermal therapy in NIR-II biowindow. B) TEM images of PdNS with edge length of B1) $50 \mathrm{~nm}$ and B4) $20 \mathrm{~nm}$, PdNS/PAA JNPs B2) $50 \mathrm{~nm}$ PdNS and B5) $20 \mathrm{~nm}$ PdNS, PdNS/ZIF-8 JNPs B3) $50 \mathrm{~nm}$ PdNS and B6) $20 \mathrm{~nm}$ PdNS. Inset in (B3) is the corresponding SEM image of a PdNS/ZIF-8 JNP. Inset in (B4) is the enlarged HR-TEM image of circled area of a PdNS. Insets in (B5) and (B6) are the corresponding enlarged PdNS/PAA JNP and PdNS/ZIF-8 JNP, respectively.
ZIF-8 by rationally designing their compositions and discovering the key properties between them.

Local hypoxia of tumors is an important indicator of tumor growth, which is the result of imbalance of oxygen supply and demand, resulting in extremely low oxygen content in local tumor areas. ${ }^{[57,58]}$ In the course of cancer treatment, local hypoxia of the tumor has a negative impact on the effects of oxygen-dependent chemotherapy. To improve the tumor-killing effect, Zhang group has uncovered an all-in-one nano-platform for efficient chemo-photodynamic therapy of local hypoxia in tumors. ${ }^{[2]}$ This multifunctional nanoplatform mounts $\mathrm{MnO}_{2}$ nanodots onto a ZIF-8 carrier that has encapsulated $g-\mathrm{C}_{3} \mathrm{~N}_{4}$ and doxorubicin hydrochloride, donated as FMZ/DC (Figure 7). The loaded $\mathrm{MnO}_{2}$ nanodots react with endogenous acidic $\mathrm{H}_{2} \mathrm{O}_{2}$ to increase the dissolved oxygen concentration for overcoming the hypoxia-caused resistance in cancer therapy, significantly improving the therapeutic efficacy of chemo-photodynamic therapy for hypoxic cancer combined with DOX and $\mathrm{C}_{3} \mathrm{~N}_{4}$. As a new nanoplatform for oxygen generation and $\mathrm{pH}$-responsive drug delivery systems, it has excellent dispersibility and satisfactory biocompatibility. Meanwhile, the $\mathrm{pH}$ sensitivity of $\mathrm{ZIF}-8$ allows quick release of the encapsulated drug in acidic $\mathrm{H}_{2} \mathrm{O}_{2}$ environment, avoiding side effects caused by nonspecific drug release of DOX. In vitro cytotoxicity experiments have shown that the designed nano-platform has a higher therapeutic effect than that without $\mathrm{MnO}_{2}$ nanodots under hypoxic conditions, or chemical and photo dynamic therapy alone with the presence of $\mathrm{MnO}_{2}$ nanodots. In vivo experiments have also demonstrated that $4 \mathrm{~T} 1$ tumors can be very effectively eliminated by the designed nanoplatform under light irradiation. In summary, the experimental results based on the $\mathrm{MnO}_{2}$ nanodots-based nanoplatform increased the oxygen concentration in the tumor microenvironment, and the vector minimized systemic toxicity, which is expected to improve the possibility of future cancer cure.

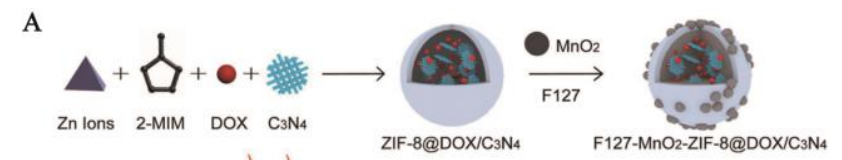

B

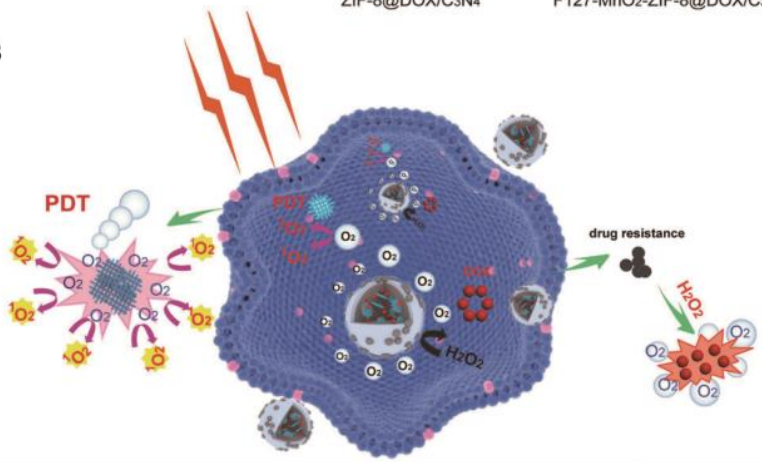

Figure 7 A) Schematic illustration of the fabrication of FMZ/DC nanocomposites. The diagram is not drawn to scale. B) Schematic illustration of FMZ/DC with oxygen generation enhancing the chemo-photodynamic therapy under $660 \mathrm{~nm}$ light irradiation.

Recently, biomimetic nanoplatform (precise treatment) have attracted wide attention for delivery of drugs through camouflage such as targeting and immune response. ${ }^{[59,60]}$ In the process of targeting tumor sites, camouflage membranes can protect drug carriers from damage caused by human immune system, prolong drug life in the blood stream and selectively target to tumors. For instance, Zou group reported a multifunc- 
tional biomimetic core-shell nanoplatform for improving synergistic chemotherapies and immunotherapies, and ZIF-8 carriers wrapped in cell membranes with good adaptability and flexible modification. ${ }^{[28]}$ ZIF-8 was used to load catalase (CAT) and doxorubicin (DOX) to construct the core for oxygen generation and drug storage (Figure 8A). The combination of the two drugs will bring about the change of $\mathrm{H}_{2} \mathrm{O}_{2}$ content, leading to the reduction of HIF-1 $\alpha$ and the increase of the effect of chemotherapy. Subsequently, the core ZIF-8 vector was encapsulated in the mouse melanoma cell membrane to improve the ability of targeting and immune escape which induced by the abundance of antigens. The morphology and size of the mZCD were observed by TEM and SEM (Figure 8B), and the average size is $130 \mathrm{~nm}$. In vivo and in vitro experiments show that this bionic core-shell nanoplatform with oxygen generation can partially accumulate in tumors. Meanwhile, with the release of drug molecule, the level of oxygen increases that further improves the efficacy of chemotherapy. Moreover, the nanocarrier has a strong effect in prolonging the recurrence time and inhibiting the metastasis of tumors. In addition, the biomineral strategy provides these two drug molecules with protection of ZIF-8 and cell membrane, which exert potential synergistic chemotherapy and immunotherapy for feasible, accurate and efficient diagnosis of cancer.
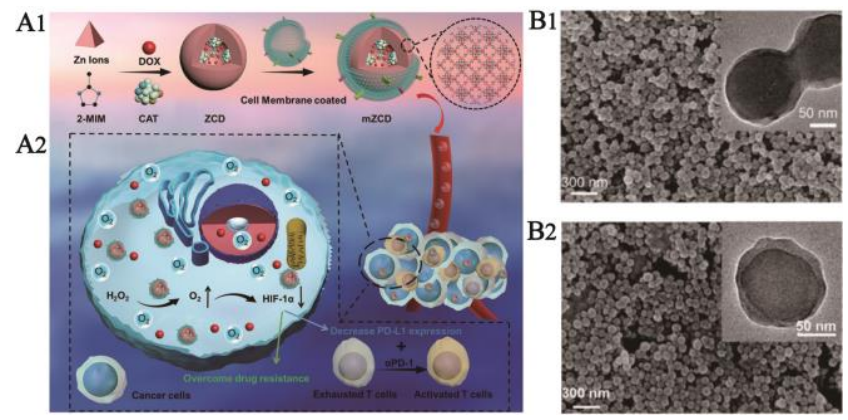

Figure 8 A) Schematic illustration of mZCD as an oxygen generating biomimetic nanoplatform for tumor chemothrapy and immunotherapy. A1) The preparation processes of mZCD. A2) The combined chemotherapy and immuotherapy of tumor. B) The characterizations of mZCD. B1) SEM image and TEM image (inserted) of mZCD. B2) SEM image and TEM image (inserted) of mZD.

In order to reduce the removal of nanoparticles by the immune system, different cell membranes have been widely used to coat nanoparticles, especially erythrocyte membrane ${ }^{[61-64]}$ Using the enhanced permeability and retention (EPR) effect of target tumor tissues, the combination of nanoparticles and cell membranes solve the problems caused by inefficiency of drug delivery and poor therapeutic effect. Zhang and co-workers reported a biomimetic nanoreactor-TGZ@eM (TPZ-GOx-ZIF-8@eM), encapsulating ZIF-8 with erythrocyte membrane (eM) as core-shell structure (Figure 9A). ZIF-8 was used as nanocarrier to encapsulate $\mathrm{GOx}$ and prodrug telazamin (TPZ). ${ }^{[29]}$ Among them, glucose oxidase $\left(\mathrm{GO}_{x}\right)$ can effectively consume endogenous glucose and oxygen, which further starves tumor cells. The anoxic microenvironment caused by oxygen consumption can transform prodrug TPZ into cytotoxic free radicals, inducing cancer cell death. Interestingly, the erythrocyte membrane shows good biomimetic properties of the outer coating, and TGZ@eM has a good modification effect, which can effectively accumulate in tumor tissues. In vivo experiments show that TGZ@eM has good selectivity and a high therapeutic effect. Importantly, it can be degraded in vivo and show sustainable release of drug molecules. The CLSM images exhibit obvious green fluorescence in the internal and edge of MCTS after treatments of GZ@eM and TGZ@eM due to the continuous $\mathrm{O}_{2}$ consumption by $\mathrm{GO}_{x}$ (Figure 9B). More importantly, this nanoparticle-based chemical curative strategy has no obvious side effect on normal organs and has great potential in the treatment of cancer in vivo. In conclusion, biomimetic nanoreactor not only explores the starvation of cancer cells caused by the loss of endogenous glucose and oxygen, but also creates a potential clinical application of colon cancer treatment strategy.

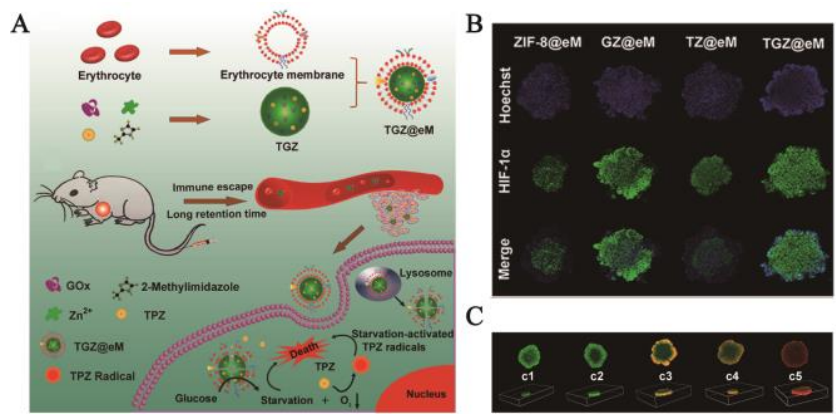

Figure 9 A) Schematic illustration of the preparation of the TGZ@eM nanoreactor and erythrocyte membrane cloaked MOF biomimetic nanoreactor for starvation-activated colon cancer therapy. B) HIF-1 $\alpha$ staining of MCTS in different groups, blue fluorescence of Hoechst 33342, green immunofluorescence staining of HIF- $1 \alpha$ antibody. C) CLSM images of MCTS incubated with PBS, ZIF-8@eM, GZ@eM, TZ@eM, and TGZ@ eM.

Biomedical imaging is an important tool in clinical medical diagnosis. ${ }^{[65,66]}$ In the diagnosis of cancer, medical imaging tools mainly contains magnetic resonance (MR), X-ray computed tomography (CT) and optical imaging. Yang and co-workers designed a multifunctional composite platform ( $\left.\mathrm{CoFe}_{2} \mathrm{O}_{4} @ P D A @ Z I F-8\right)$ consisting of a mesoporous core $\mathrm{CoFe}_{2} \mathrm{O}_{4}$ and a shell ZIF-8. ${ }^{[67]}$ The average diameters of flower-like structure of $\mathrm{CoFe}_{2} \mathrm{O}_{4}, \mathrm{CoP}$ and CoPZ MNPs are 70, 100 and $150 \mathrm{~nm}$, respectively (Figure 10A). The nanoplatform exhibited enhanced tumor diagnosis and treatment efficiency by combining nuclear magnetic imaging, chemotherapy, and photothermal therapy, in which the mesoporous core is used as a T2-MR imaging probe and the core-shell vector is loaded with two anticancer drugs (hydrophilic DOX and hydrophobic CPT), respectively. An infrared thermal imaging camera was used to record the change of photothermal capacity intuitively. A temperature increase of nearly $20^{\circ} \mathrm{C}$ have been observed in vivo infrared thermal photographs of $\mathrm{Co} / \mathrm{DPZ} / \mathrm{C}$ within $10 \mathrm{~min}$ under the irradiation of $808 \mathrm{~nm}$ laser (Figure 10B). In addition, the mesoporous core $\mathrm{CoFe}_{2} \mathrm{O}_{4}$ was modified with a dobutamine (PDA) layer to avoid DOX leakage. The experimental results show effective drug releasing under the dual stimulation of near-infrared illumination and tumor micro-acid environment. It is worth noting that synergistic treatment of magnetic resonance imaging-guided chemotherapy and PTT significantly improve the anticancer effect with satisfactory biocompatibility. In addition, the simple multi-functional composite system overcomes the limitations of the multi-drug administration strategy and achieves the combined use of the hydrophilic and hydrophobic drugs. Together with its excellent photothermal performance and controllable multi-drug release, it can be used as an effective platform for diagnosis, intelligent drug delivery and cancer treatment.

In order to fully exploit the advantages of various imaging technologies and avoid the insufficiency of single imaging technology, the development of multi-modal imaging technology has become an important part of the field of biomedica 

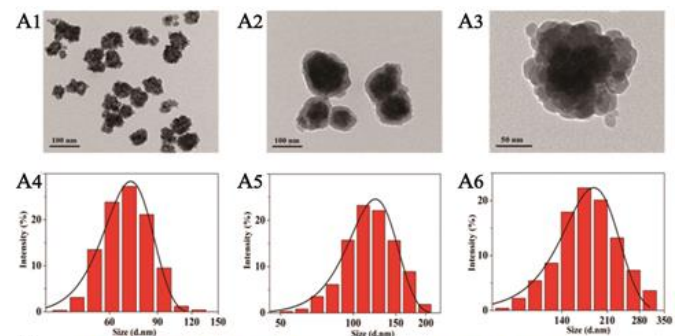

B
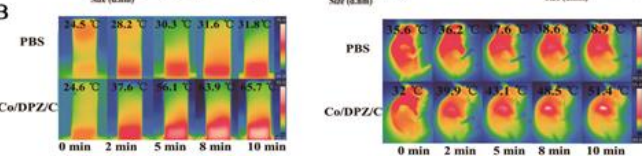

Figure 10 (A1-A3) TEM images and (A4-A6) DLS size distributions of $\mathrm{CoFe}_{2} \mathrm{O}_{4}, \mathrm{CoP}$, and $\mathrm{CoPZ}$. $\mathrm{B}$ ) In vitro and in vivo infrared thermal photographs of PBS and Co/DPZ/C exposed to $808 \mathrm{~nm}$ laser recorded at different time intervals.

imaging. ${ }^{[68-70]}$ Wang group reported the use of multifunctional $\mathrm{Fe}_{3} \mathrm{O}_{4} @$ poly(acrylic acid)/Au nanoclusters/ZIF-8 NPs $\left(\mathrm{Fe}_{3} \mathrm{O}_{4} @\right.$ PAA/AuNCs/ZIF-8) for cancer diagnosis and visualization-synergistic therapy. ${ }^{[71]}$ Firstly, $\mathrm{Fe}_{3} \mathrm{O}_{4}$ NPs were modified by CTAB to produce $\mathrm{Fe}_{3} \mathrm{O}_{4} @ P A A$ core-shell NPs under ultrasonic. Subsequently, AuNCs were added to the $\mathrm{Fe}_{3} \mathrm{O}_{4} @ P A A$ nanoparticles, and a ZIF-8 shell was formed on the surface to support DOX. Figure 11A shows morphological changes with sequential modification of nanomedicine, $\mathrm{Fe}_{3} \mathrm{O}_{4} @ P A A / A u N C s /$ ZIF-8 core-shell NPs exhibited flower-like shape with a diameter of $130 \mathrm{~nm}$. In vitro and in vivo experiments showed that the obtained $\mathrm{Fe}_{3} \mathrm{O}_{4} @ \mathrm{PAA} / \mathrm{Gncs} / \mathrm{ZIF}-8 \mathrm{NPs}$ had dual pH-responsive drug release and high DOX loading capacity, while the nanoparticles ensured three-mode imaging ability and effective tumor suppression. The CT signal intensity enhances visibly with the increasing concentration of the NPs while the T2 signal intensity reduces gradually. Strong orange fluorescence emitted by $\mathrm{Au} \mathrm{NCs}$ and the corresponding bright-field fluorescence microscope image from the $\mathrm{Fe}_{3} \mathrm{O}_{4} @ \mathrm{PAA} / \mathrm{AuNCs} / \mathrm{ZIF}-8 \mathrm{NPs}$ is displayed in the cytoplasm regions of the cells (Figure 11B). In summary, trimodal imaging can circumvent the limitations of different imaging techniques and show great potential in cancer therapy and visual synergistic therapy.
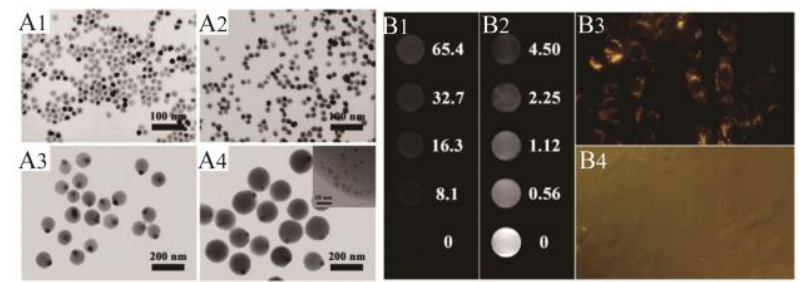

Figure 11 A) TEM images of (A1) OA-modified $\mathrm{Fe}_{3} \mathrm{O}_{4} \mathrm{NPs}$, (A2) CTAB-stabilized $\mathrm{Fe}_{3} \mathrm{O}_{4} \mathrm{NPs}$, (A3) $\mathrm{Fe}_{3} \mathrm{O}_{4} @ P A A$ core-shell $\mathrm{NPs}$, and (A4) $\mathrm{Fe}_{3} \mathrm{O}_{4} @ \mathrm{PAA} / \mathrm{AuNCs} / \mathrm{ZIF}-8$ core-shell NPs; B1) CT phantom images and B2) MR images of HepG-2 cells incubated with $\mathrm{Fe}_{3} \mathrm{O}_{4} @ P A A / A u N C s / Z I F-8$ NPs. B3) CLSM image

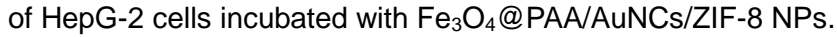
B4) Corresponding bright-field contrast image.

\section{Conclusion}

This article details the mechanism of ZIF-8 as a drug carrier and its advantages in the delivery of anticancer drugs. As outlined, the ZIF-8 nanoplatform has been widely used in nanomedicine, from single-functional system to multi-functional ZIF-8 nanoplatform. Importantly, combined with the unique structure and superior biocompatibility of ZIF-8, the versatile platform and delivery system can be easily and quickly manu- factured for packaging functional proteins, chemotherapeutic drugs, DNA and other small fractions of drugs, and achieving excellent performances in anti-cancer or other diseases.

Meanwhile, these systems have also many challenges to be overcome. The demand for bio-applications is not only a problem of cancer treatment, but also a solution to the biocompatibility, biodegradability and stability of ZIF-8. Firstly, the biotoxicity of nanocomposites is a basic concern for clinical applications. The prepared nanocarrier should be safe without harming normal cells and tissues, as well as body metabolism. Meanwhile, the immune response and tissue repair process in the body is very complicated. The fabricated nano-medicine should escape from the guard of immune systems and achieving long-time blood circulation. Therefore, the bio-description of ZIF-8 nanoparticles should be clear not only the nature of the material itself, but also the interaction between the nanoparticles and the local environment. Problems with the interaction with the body environment require further systematic investigation. In addition, the biodegradability and stability of ZIF-8 is a dynamic process in all types of environments, not static. Although researchers have discussed the degradation process and stability of ZIF-8 under different conditions, the fundamental picture of metabolic pathway of nanomedicine has still been masked. Therefore, a large number of studies should be carried out under simulated physiological conditions, especially, simulating how the drug-loading platform delivers various types of drugs to the target area and further monitor the drug release process in the body.

Despite significant advances and potential clinical application in cancer treatment, the use of ZIF-8 for clinical applications remains a long-term challenge in the development of nanomedicine. Simultaneously, considering the excellent performance of synergistic effect of different treatment strategies, the ZIF-8-based multi-functional treatment platform has also been one of the main directions for future efforts.

\section{Author Contributions}

The manuscript was written through contributions of all authors. All authors have given approval to the final version of the manuscript.

\section{Acknowledgments}

This project was financially supported by the National Key R\&D Program of China (No. 2016YFA0201200), the National Science Foundation for Young Scientists of China (No. 21807069), Shanghai Outstanding Technology Leader (No. 19XD1431900) and Shanghai Rising-Star Program (No. 17QB1402900)

\section{References}

[1] Chen, B.; Yang, Z.; Zhu, Y.; Xia, Y. J. Mater. Chem. A 2014, 2, 16811.

[2] Wang, B.; Côté, A. P.; Furukawa, H.; O’Keeffe, M.; Yaghi, O. M. Nature 2008, 453, 207.

[3] Phan, A.; Doonan, C. J.; Uribe-Romo, F. J.; Knobler, C. B.; O’keeffe, M.; Yaghi, O. M. Acc. Chem. Res. 2009, 43, 58.

[4] Park, K. S.; Ni, Z.; Côté, A. P.; Choi, J. Y.; Huang, R.; Uribe-Romo, F. J.; Chae, H. K. Proc. Natl. Acad. Sci. U. S. A. 2006, 103, 10186.

[5] Huang, X. C.; Lin, Y. Y.; Zhang, J. P. Angew. Chem. Int. Ed. 2006, 45, 1557.

[6] Cravillon, J.; Münzer, S.; Lohmeier, S. J.; Feldhoff, A.; Huber, K. Chem. Mater. 2009, 21, 1410.

[7] Nune, S. K.; Thallapally, P. K.; Dohnalkova, A.; Wang, C. Chem. Commun. 2010, 46, 4878.

[8] Venna, S. R.; Jasinski, J. B.; Carreon, M. A. J. Am. Chem. Soc. 2010, 132, 18030. 
[9] Ameloot, R.; Gobechiya, E.; Ujii, H.; Martens, J. A.; Hofkens, J. Adv. Mater. 2010, 22, 2685.

[10] Cravillon, J.; Nayuk, R.; Springer, S.; Feldhoff, A.; Huber, K. Chem. Mater. 2011, 23, 2130.

[11] Zhuang, J.; Kuo, C. H.; Chou, L. Y.; Liu, D. Y.; Weerapana, E.; Tsung, C.-K., ACS Nano 2014, 8, 2812.

[12] Lu, G.; Li, S.; Guo, Z.; Farha, O. K.; Hauser, B. G.; Qi, X.; Wang, Y.; Wang, X. Nat. Chem. 2012, 4, 310.

[13] Bustamante, E. L.; Fernández, J. L.; Zamaro, J. M. J. Colloid Interface Sci. 2014, 424, 37.

[14] Horcajada, P.; Chalati, T.; Serre, C.; Gillet, B.; Sebrie, C.; Baati, T.; Eubank, J. F. Nat. Mater. 2010, 9, 172.

[15] Wang, Y.; Zhang, W.; Guo, J.; Duan, W.; Liu, B. ACS Appl. Mater. Interfaces 2019, 11, 38016

[16] McKinlay, A. C.; Morris, R. E.; Horcajada, P.; Férey, G.; Gref, R.; Couvreur, P.; Serre, C. Angew. Chem. Int. Ed. 2010, 49, 6260.

[17] Vasconcelos, I. B.; da Silva, T. G.; Militão, G. C.; Soares, T. A.; Rodrigues, N. M.; Rodrigues, M. O.; da Costa, N. B.; Freire, R. O. RSC Adv. 2012, 2, 9437.

[18] Meek, S. T.; Greathouse, J. A.; Allendorf, M. D. Adv. Mater. 2011, 23, 249.

[19] Zhou, W.; Zou, B.; Zhang, W.; Tian, D.; Huang, W.; Huo, F. Nanoscale 2015, 7, 8720 .

[20] Ettlinger, R.; Moreno, N.; Volkmer, D.; Kerl, K.; Bunzen, H. Chem. Eur. J. 2019, 25, 13189

[21] Chen, T. T.; Yi, J. T.; Zhao, Y. Y.; Chu, X. J. Am. Chem. Soc. 2018, 140, 9912.

[22] Zhang, H.; Li, Q.; Liu, R.; Zhang, X.; Li, Z.; Luan, Y. Adv. Funct. Mater. 2018, 28, 1802830

[23] Zhou, W.; Wang, L.; Li, F.; Zhang, W.; Huang, W. Adv. Funct. Mater 2017, 27, 1605465

[24] Yang, C.; Xu, J.; Yang, D.; Wang, X.; Liu, B.; He, N.; Wang, Z., Chin. Chem. Lett. 2018, 29, 1421.

[25] Wang, X.; Li, X.; Liang, X.; Liang, J.; Zhang, C.; Yang, J. J. Mater. Chem. $B$ 2018, 6, 1000

[26] Zhang, L.; Li, S.; Chen, X.; Wang, T.; Li, L.; Su, Z.; Wang, C. Adv. Funct. Mater. 2018, 28, 1803815

[27] Zhang, W.; Li, S.; Liu, X.; Yang, C.; Hu, N.; Dou, L.; Zhao, B. Adv. Funct. Mater. 2018, 28, 1706375.

[28] Zou, M. Z.; Liu, W. L.; Li, C. X.; Zheng, D. W.; Zeng, J. Y.; Gao, F. Small 2018, 14, e1801120.

[29] Zhang, L.; Wang, Z.; Zhang, Y.; Cao, F.; Dong, K.; Ren, J.; Qu, X. ACS Nano 2018, 12, 10201.

[30] Chen, X.; Tong, R.; Shi, Z.; Yang, B.; Liu, H.; Ding, S. ACS Appl. Mater. Interfaces 2018, 10, 2328.

[31] Alsaiari, S. K.; Patil, S.; Alyami, M.; Alamoudi, K. O.; Aleisa, F. A.; Merzaban, J. S.; Li, M.; Khashab, N. M., Journal of the American Chemical Society 2018, 140, 1, 143

[32] Xu, D.; You, Y.; Zeng, F.; Wang, Y.; Liang, C. ACS Appl. Mater. Interfaces 2018, 10, 15517

[33] Zhang, Y.; Wang, F.; Ju, E.; Liu, Z.; Chen, Z.; Ren, J.; Qu, X., Adv. Funct. Mater. 2016, 26, 6454.

[34] Liang, K.; Ricco, R.; Doherty, C. M.; Styles, M. J.; Bell, S.; Kirby, N.; Mudie, S.; Haylock, D.; Hill, A. J.; Doonan, C. J. Nat. Commun. 2015, 6, 7240 .

[35] Larrañeta, E.; Henry, M.; Irwin, N. J.; Trotter, J. Carbohydrate Polymers 2018, 181, 1194.

[36] Bhatnagar, P.; Pant, A. B.; Shukla, Y.; Panda, A. Eur. J. Pharm. Biopharm. 2016, 105, 176.

[37] Yahiro, J.; Kawano, T.; Imai, H. J. Colloid Interface Sci. 2007, 310, 302.

[38] Selegård, R.; Aronsson, C.; Brommesson, C.; Dånmark, S.; Aili, D. Sci. Rep. 2017, 7, 7013

[39] Ren, H.; Zhang, L.; An, J.; Wang, T.; Li, L.; Si, X.; He, L.; Wu, X. Chem. Commun. 2014, 50, 1000

[40] Sun, C. Y.; Qin, C.; Wang, X. L.; Yang, G. S.; Shao, K. Z.; Lan, Y. Q.;
Su, Z. M.; Huang, P.; Wang, C. G.; Wang, E. B. Dalton Trans. 2012 41, 6906.

[41] Lyu, F.; Zhang, Y.; Zare, R. N.; Ge, J.; Liu, Z. Nano Lett. 2014, 14 5761.

[42] Liang, K.; Richardson, J. J.; Doonan, C. J.; Mulet, X.; Ju, Y.; Cui, J.; Caruso, F.; Falcaro, P. Angew. Chem. Int. Ed. 2017, 56, 8510.

[43] Erazo-Oliveras, A.; Najjar, K.; Dayani, L.; Wang, T.-Y.; Johnson, G. A. Nat. Methods 2014, 11, 861 .

[44] Leader, B.; Baca, Q. J.; Golan, D. E. Nat. Rev. Drug Discov. 2008 7, 21.

[45] Gu, Z.; Biswas, A.; Zhao, M.; Tang, Y. Chem. Soc. Rev. 2011, 40, 3638.

[46] Zheng, H.; Zhang, Y.; Liu, L.; Wan, W.; Guo, P. J. Am. Chem. Soc. 2016, 138, 962.

[47] Kaur, J.; Singh, G.; Saini, S.; Rana, A. J. Drug Deliv. Therap. 2012, $2,16$.

[48] Li, Y.; Jin, J.; Wang, D.; Lv, J.; Hou, K.; Liu, Y.; Chen, C.; Tang, Z. Nano Res. 2018, 11, 3294.

[49] Gomar, M.; Yeganegi, S. Micropor. Mesopor. Mat. 2017, 252, 167.

[50] Tung, N.-T.; Tran, C.-S.; Nguyen, H.-A.; Nguyen, T.-D.; Chi, S.-C.; Pham, D.-V.; Bui, Q.-D.; Ho, X.-H. Int. J. Pharm. 2019, 555, 63.

[51] Ho, D.K.; Nichols, B. L.; Edgar, K. J. Eur. J. Pharm. Biopharm. 2019, 144, 110

[52] Xiao, D.; Jia, H. Z.; Zhang, J.; Liu, C. W.; Zhuo, R. X.; Zhang, X. Z. Small 2014, 10, 591.

[53] Feng, Y.; Wang, H.; Zhang, S.; Zhao, Y.; Gao, J.; Zheng, Y.; Zhao, P.; Zhang, Z.; Zaworotko, M. J.; Cheng, P. Adv. Mater. 2019, 31 1805148

[54] Martin, N.; Ma, D.; Herbet, A.; Boquet, D.; Winnik, F. o. M.; Tribet, C. Biomacromolecules 2014, 15, 2952.

[55] Wang, D.; Wu, H.; Zhou, J.; Xu, P.; Wang, C.; Shi, R.; Wang, H. Adv. Sci. 2018, 5, 1800287.

[56] Li, X.; Zhou, L.; Wei, Y.; El-Toni, A. M. J. Am. Chem. Soc. 2014 136, 15086.

[57] Gao, S.; Wang, G.; Qin, Z.; Wang, X.; Zhao, G.; Ma, Q.; Zhu, L. Biomaterials 2017, 112, 324.

[58] Chen, J.; Keltner, L.; Christophersen, J.; Zheng, F.; Krouse, M., The Cancer Journal 2002, 8, 2, 154.

[59] Lin, E. P. Y.; Yang, C. Y.; Lin, C. W.; Huang, B. T.; Lai, W. Y.; Tseng, Y. T.; Yang, P. C. J. Clin. Oncol. 2017, 35, e20087.

[60] Wang, C.; Wang, J.; Zhang, X.; Yu, S.; Wen, D.; Hu, Q. Sci. Transl. Med. 2018, 10, eaan3682.

[61] Ding, H.; Lv, Y.; Ni, D.; Wang, J.; Tian, Z.; Wei, W.; Ma, G., Nanoscale 2015, 7, 9806.

[62] Pei, Q.; Hu, X.; Zheng, X.; Liu, S.; Li, Y.; Jing, X.; Xie, Z. ACS Nano 2018, 12, 1630.

[63] Xuan, M.; Shao, J.; Zhao, J.; Li, Q. Angew. Chem. Int. Ed. 2018, 57, 6049.

[64] Gao, W.; Hu, C. M. J.; Fang, R. H.; Luk, B. T.; Su, J.; Zhang, L., Adv. Mater. 2013, 25, 3549.

[65] Yao, C.; Wang, P.; Li, X.; Hu, X.; Hou, J.; Wang, L.; Zhang, F. Adv. Mater. 2016, 28, 9341

[66] Li Volsi, A.; Scialabba, C.; Vetri, V.; Cavallaro, G. ACS Appl. Mater. Interfaces 2017, 9, 14453

[67] Yang, J. C.; Chen, Y.; Li, Y. H.; Yin, X. B. ACS Appl. Mater Interfaces 2017, 9, 22278.

[68] Lee, N.; Hyeon, T. Chem. Soc. Rev. 2012, 41, 2575

[69] Michaelis, J.; Hettich, C.; Mlynek, J.; Sandoghdar, V. Nature 2000 405, 325

[70] Na, H. B.; Song, I. C.; Hyeon, T. Adv. Mater. 2009, 21, 2133.

[71] Bian, R.; Wang, T.; Zhang, L.; Li, L.; Wang, C. Biomater. Sci. 2015, 3, 1270 .

Received October 29, 2019

Accepted November 21, 2019 